\title{
BMJ Open Nasogastric/Nasoenteric tube-related incidents in hospitalised patients: a study protocol of a multicentre prospective cohort study
}

\author{
Fernanda Raphael Escobar Gimenes, ${ }^{\oplus 1}$ Marta Cristiane Alves Pereira, ${ }^{1}$ \\ Patricia Rezende do Prado, ${ }^{2}$ Rhanna Emanuela Fontenele Lima de Carvalho, ${ }^{3}$ \\ Janine Koepp, ${ }^{4}$ Ligia Menezes de Freitas, ${ }^{5}$ Thalyta Cardoso Alux Teixeira, ${ }^{6}$ \\ Adriana Inocenti Miasso ${ }^{7}$
}

To cite: Gimenes FRE, Pereira MCA, Prado PRdo, et al. Nasogastric/Nasoenteric tuberelated incidents in hospitalised patients: a study protocol of a multicentre prospective cohort study. BMJ Open 2019;9:e027967. doi:10.1136/ bmjopen-2018-027967

- Prepublication history for this paper is available online. To view these files, please visit the journal online (http://dx.doi. org/10.1136/bmjopen-2018027967).

Received 18 November 2018 Revised 26 June 2019 Accepted 1 July 2019
D) Check for updates

(c) Author(s) (or their employer(s)) 2019. Re-use permitted under CC BY-NC. No commercial re-use. See rights and permissions. Published by BMJ.

For numbered affiliations see end of article.

\section{Correspondence to} Dr Fernanda Raphael Escobar Gimenes; fregimenes@eerp.usp.br

\section{ABSTRACT}

Introduction Hospitalised patients with nasogastric/ nasoenteric tube (NGT/NET) are at constant risk of incidents; therefore, healthcare professionals need to routinely monitor risks and adopt strategies for patient safety and quality of care.

Aim This study aimed to evaluate the NGT/NET-related incidents in hospitalised patients and associated factors. Methods This is a multicentre study, with a prospective cohort design. Data will be collected at the general medical ward of seven Brazilian hospitals in the north, northeast, southeast and south. The sample will consist of 391 patients that require an NGT/NET during hospitalisation. Three different methods will be used to identify the incidents: (1) healthcare professionals and patients/caregivers will be required to report any NGT/ NET-related incidents; (2) researchers will visit the wards to get information about the incidents with healthcare professionals and patients/caregivers; (3) the researchers will review the medical records looking for information on the occurrence of any NGT/NET-related incidents. Demographic, clinical and therapeutic details will be obtained from the medical records and will be registered in an electronic data collection tool developed for the purposes of this study. The complexity of patients will be assessed by the Patient Classification System, and the severity of comorbid diseases will be assessed through the Charlson Comorbidity Index.

Implication for practice The results may encourage the use of evidence effectively to influence the scientific foundation for clinical practice and the development of evidence-based policies that will prevent, manage and eliminate complications caused by NGT/NET-related incidents, and improve the quality and safety of care provided to hospitalised patients.

Ethics and dissemination The study has been approved by the Research Ethics Committee. Detailed information about the study can be provided by the principal investigator. The findings will be reported through academic journals, seminar and conference presentations, social media, print media, the internet and community/ stakeholder engagement activities.
Strengths and limitations of this study

- Several single-centre studies have identified the prevalence of nasogastric/nasoenteric tube (NGT/ NET)-related incidents in general inpatients; however, there are no studies reporting on those incidents across multiple hospital sites and at a national level.

- A multicentre study may ascertain better generalisability of the data.

- To our knowledge, this is the first large-scale study in Brazil and in Latin America documenting the incidence of NGT/NET-related incidents in internal medicine wards.

- Although voluntary reporting can detect a broad range of incidents, this system misses the vast majority of incidents and cannot provide stable estimates of the true underlying causes.

- It is difficult to demonstrate causality between NGT/ NET-related incidents and negative patient outcomes due to a number of confounding factors.

\section{INTRODUCTION}

During hospitalisation, patients with advanced disease have caloric and protein malnutrition, and require nutritional and drug interventions. Therefore, these patients often need a nasogastric/nasoenteric tube (NGT/NET) to ensure that their daily nutritional and medical needs are met. ${ }^{1}$ Data from the American Society for Enteral and Parenteral Nutrition revealed that more than 245000 patients per year require at least a temporary feeding tube during a hospital stay, along with another nearly 31000 patients that are on enteral nutrition at home. ${ }^{2}$ In the USA, about one million NGT/NET are introduced in adults and children each year, ${ }^{3}$ and the rate of complications is $2 \%-36 \%{ }^{4-6}$

Although inserting an NGT/NET is a relatively innocuous procedure, improper 
positioning can cause severe and fatal complications. ${ }^{7-9}$ One of the major incidents related to NGT/NET is the wrong connection. The design of these devices is such that it is possible to infuse enteral feeding and/or medications via an unwanted route, such as intravenous route. ${ }^{1011}$

It is also common to have inadvertent positioning of the tube in the respiratory tract, resulting in bronchoaspiration and pneumothorax. ${ }^{8}{ }^{12-14}$ Pulmonary aspiration occurs in $2 \%-10 \%$ of patients receiving enteral feeding. The inadvertent insertion of the tube into the tracheal tree results in discomfort for the patient, delayed feeding, increased morbidity and mortality, and length of hospital stay, resulting in added burdens for the institution and patients. ${ }^{5} 15$ Therefore, healthcare professionals need to routinely monitor risks and adopt strategies aimed at patient safety.

Other incidents may occur during the insertion and/ or progression of an NGT/NET through the gastrointestinal tract, such as sinusitis, vomiting, nasopharyngeal discomfort, erosion of the nasal septum, epistaxis and blood return by the tube during guidewire withdrawal. ${ }^{16}$ In Brazil, these data are not available; however, it is observed that NGT/NET are a common procedure in most Brazilian health institutions. ${ }^{17}$

Regarding medication administration, administering drugs with absorption at the intestinal level in patients using an NGT is a challenge. As demonstrated in the literature, some drugs should never be crushed and administered through an NGT because they have enteric or controlled release protection coatings. ${ }^{18}$ The grinding process of these drugs can destroy the coating film and result in incidents, exposing patients to unnecessary risks. ${ }^{19}$ In this regard, experts argue that healthcare professionals should not assume that a drug formulated to be administered orally can be administered safely through an NGT/NET because drug delivery mechanisms can be altered or destroyed, reducing effectiveness or increasing risk of toxicity. ${ }^{20}$

In order to achieve adequate clinical outcomes in hospitalised patients, it is essential that medication and enteral nutrition be administered appropriately. However, nursing guidelines on enteral feeding tube care are not based on scientific evidence, but on traditions, rituals and expert opinions, exposing patients to unnecessary harm. ${ }^{21}$

Although NGT/NET-related incidents are common in hospital settings with significant morbidity and mortality, the issue has not been extensively studied, especially in developing countries. Given that the number of patients with chronic conditions have increased significantly worldwide, as well as in Brazil, it is critical that healthcare professionals employ a repertoire of evidence based on interventions in order to provide safe and high quality care. ${ }^{22}{ }^{23}$ However, there is a knowledge gap regarding the safe handling of NGT/NET in Brazilian healthcare institutions. Studies that aim to identify the most frequent NGT/NET-related incidents that occur during hospital stays can reduce that gap and the risks of complications and lower overall cost of care.
The results of the study will contribute to the production of knowledge and its application in clinical practice for harm reduction towards a safer environment for patient care, will provide national and international data that reflect the problem of NGT/NET-related incidents in acute care units and will contribute to the use of the evidence in an efficient way to influence the development of national practices and policies aimed at managing the risks associated with the NGT/NET.

\section{Significance and theoretical framework}

Feeding tubes may be used long term (ie, gastrostomy or jejunostomy) or short term with distal tips positioned in the stomach (ie, NGT) or small intestine (ie, NET) ${ }^{24}$ Long-term feeding tubes are not part of the scope of this study, and therefore, these enteral access devices will not be addressed.

These devices are not exclusively used for administration of enteral nutrition. They are also frequently used for administering drugs. The consequence of this duo use is the increased risk of tube obstructions, physical-chemical incompatibilities and potential drug-nutrient interactions. ${ }^{25}$ In a previous study, researchers found that $74 \%$ of nurses employed wrong medication administration methods to deliver medicines through NGT/NET and those errors could reduce the effects of drugs and lead to unsuccessful treatments. ${ }^{26}$

According to a study conducted in a Brazilian private hospital, the main reason for the loss of an NGT/NET was obstruction $(36 \%)$ related to wrong medication preparation and administration techniques. ${ }^{27}$ Estimates of incidence of clogged feeding tubes range widely from $12.5 \%$ to $45 \%$, but it is undisputed that they result in increased costs for patients and institutions. ${ }^{28}$ Thus, healthcare practitioners, especially nurses, should not assume that a medication intended to be taken by mouth can be safely administered through an NGT/NET because this misconception can result in harm to patients and increase medical costs to society. ${ }^{21}$

Another incident related to improper medication administration via an NGT/NET is caused by drug-drug interactions in older people due to polypharmacy, with a prevalence of $20 \%-40 \%$. Polypharmacy increases the complexity of clinical management and contributes to medication adverse events. ${ }^{29}$

Therefore, the causes of the incidents are multifaceted and the consequences are associated with increasing complexity of patients and treatments, intensifying the need for measures aimed at improving the quality of care. For this reason, opportunities to share knowledge are fundamental to patient safety. ${ }^{30}$

Efforts to overcome these problems have resulted in the development of international programmes and policies. For instance, the WHO proposed the patient safety research cycle and the first step in improving care quality and safety is to measure the occurrence of incidents in order to know the magnitude of the problem. The WHO 
then suggests understanding the causes and identifying solutions. ${ }^{31}$

In Brazil, the Ministry of Health and the National Health Surveillance Agency, in line with WHO's global initiatives, launched the National Patient Safety Program in mid-April 2013 with the aim of promoting strategies aimed at prevention and reduction of risks associated with healthcare. Among the strategies were increasing patient safety research. ${ }^{3233}$

The present study is necessary when considering the fact that patient safety is a global problem affecting countries at all levels of development ${ }^{34}$; the production of knowledge and its application in clinical practice can promote a safe environment for the provision of care, and in Brazil, there is concern about the need to increase knowledge on this issue and there are insufficient national data that reflect the problem of the incidents related to NGT/NET in hospitalised patients. We expect that the results of this study will provide high quality evidence that will support interventions designed to improve patient safety.

The objectives of this study are to evaluate the NGT/ NET-related incidents in hospitalised patients and associated factors. The secondary objectives are as follows:

1. To analyse the association between the NGT/NETrelated incidents and the complexity of the patients.

2. To analyse the association between the NGT/NET-related incidents and the severity of comorbid diseases.

3. To examine the predictive role of Charlson Comorbidity Index (CCI) on mortality of patients with an NGT/ NET.

4. To assess potential drug-drug interactions (pDDIs) in patients with an NGT/NET and its association with patient complexity and comorbid diseases.

\section{METHODS}

\section{Research design}

This is a multicentre study, with a prospective cohort design.

\section{Setting}

Seven centres across Brazil will participate in this study; the centres include a mix of community and university hospitals, hospitals with and without residency programmes, and public and private hospitals; they also vary in size. The hospitals will be as follows: Acre Hospital of Clinics (HCA), General Hospital of Fortaleza (HGF), Hospital of Clinics of the Medical School of Ribeirão Preto of the University of São Paulo (HCFMRP-USP), Américo Brasiliense State Hospital (HEAB), Sumaré State Hospital (HES), São Vicente de Paulo Hospital (HSVP) and Santa Cruz Hospital (HSCRGS).

The medical wards of these hospitals were chosen for this study because many adult patients in these wards have chronic conditions and require enteral nutrition and medications through an NGT/NET.

\begin{tabular}{lclc|}
\hline Table 1 & Determination of sample size, 2018 \\
\hline Hospital/Strata & Inpatients, $\mathbf{n}$ & $\begin{array}{l}\text { Prevalence of } \\
\text { patients with } \\
\text { NGT/NET }\end{array}$ & $\begin{array}{l}\text { Estimated } \\
\text { size of each } \\
\text { stratum }\end{array}$ \\
\hline HCA & 980 & 0.1908 & 58 \\
HGF & 192 & 0.1563 & 11 \\
HCFMRP-USP & 2247 & 0.0788 & 134 \\
HEAB & 783 & 0.1073 & 47 \\
HES & 1991 & 0.1828 & 119 \\
HSVP & 323 & 0.0650 & 19 \\
\hline HSCRGS & 48 & 0.0324 & 3 \\
\hline
\end{tabular}

HCA, Acre Hospital of Clinics; HCFMRP-US, Hospital of Clinics of the Medical School of Ribeirão Preto of the University of São Paulo; HEAB, Américo Brasiliense State Hospital; HES, Sumaré State Hospital; HGF, General Hospital of Fortaleza; HSCRGS, Santa Cruz Hospital; HSVP, São Vicente de Paulo Hospital; NGT/NET, nasogastric/nasoenteric tube.

\section{Subjects}

The study population will be the patients admitted to the medical ward of the seven Brazilian hospitals that require a feeding tube during hospitalisation. The inclusion criteria are patients older than 18 years, who are admitted to the medical ward with a short-term feeding tube (NGT or NET), patients who required the insertion of an NGT/ NET and patients that have been hospitalised for at least 24 hours. Patients meeting the above inclusion criteria who are readmitted during the study period will be only counted for their first admission.

Sampling size was determined by a stratified random sampling with proportional allocation by strata, where each stratum is formed by the floors/units of each hospital (table 1).

The formula for the calculation of the sample size is given by:

$$
n=\frac{z_{\frac{\alpha}{2}}^{2} N(1-P)}{\varepsilon^{2} P(N-1)+z_{\frac{\alpha}{2}}^{2}(1-P)}
$$

where $P$ represents the prevalence of the event of interest

(column 3 of table 1), $z_{\frac{\alpha}{2}}$ represents the level of significance adopted and $o \varepsilon$ is the relative sampling error. If the sample size calculated by the expression given in (1) is greater than $20 \%$ of the population, the following finite correction procedure is adopted:

$$
n c=\frac{n}{\left(1+\frac{n}{N}\right)}
$$

where $N$ is the total size of the study population and $n$ is the value obtained in (1). The sample was allocated proportionally among the $\mathrm{H}$ strata according to the formula:

$$
n_{h}=n \frac{N_{h}}{N},
$$

where $N$ is the total population of hospitalised patients (n=6564) and $N_{h}$ is the total of each stratum H. Population 
totals are found in the second column of table 1. Adopting the parameters of relative error of $20 \%$, level of significance of $5 \%$ and the total population of 6564 , a total sample size of 391 patients was calculated. The required sample sizes of each strata are found in the last column of table 1.

\section{Instruments}

Data collection tools will be composed of three virtual forms which will be developed by the research team and assessed for face and content validity by a panel of experts. The forms will be developed in Portuguese and an online platform (Survey Monkey) will be used.

The experts will be selected through the analysis of existing curricula in the database of the Brazilian National Council for Scientific and Technological Development and will be invited to participate in the study, by invitation letter sent by electronic mail. Then the access links to the electronic forms will be made available for the experts to carry out their analysis; the experts will have up to 30 days to evaluate the electronic forms and give their feedback to the researchers. The modified forms will be tested through a pretest, where the forms will be applied to five hospitalised patients from the first day of use of an NGT/NET until patient discharge.

At admission, demographic, clinical and therapeutic information will be recorded in the first electronic data collection form, including

- Demographic data: registration number; date of admission to the ward/unit; date of birth; city/state of origin; gender; race; marital status; education level; profession; origin of the referral.

- Clinical data: principal and secondary International Classification of Diseases (ICD); comorbidities; the final score of the Patient Classification System $(\mathrm{PCS})^{35}$; level of consciousness measured by Glasgow Coma Scale (GCS).

- Therapeutic data: data related to the NGT/NET (time to insertion; type; gauge; location [gastric or small bowel]; methods used to confirm feeding tube placement and the results of the methods used); data related to the enteral nutrition (type, total volume in 24 hours, frequency of administration); data related to the medications prescribed (drug name; dosage form; dose; route of administration; frequency of administration; and planned/scheduled administration time).

The second electronic data collection form will include variables related to the incidents:

- Incident: date and time of occurrence; type of incident (mechanic; gastrointestinal; infectious; and others $)^{4}$; consequence of the incident for the patient, according to the WHO (none; mild; moderate; severe; and fatal) ${ }^{36}$; measures adopted after the incident; sources of information on the occurrence of the incident; and means of obtaining information about the incident.
- Clinical: principal and secondary ICD; comorbidities; the final score of the $\mathrm{PCS}^{35}$; level of consciousness measured by GCS.

- Therapeutic: data related to the enteral nutrition (type, total volume in 24 hours, frequency of administration); data related to the medications prescribed (drug name; dosage form; dose; route of administration; frequency of administration; and planned/ scheduled administration time).

The third electronic data collection form will include variables related to the start of feeding tube and patient follow-up:

- Clinical: date of the start of the feeding tube use; principal and secondary ICD; comorbidities; the final score of the $\mathrm{PCS}^{35}$; level of consciousness measured by GCS.

- Therapeutic: data related to the medications prescribed 24 hours after feeding tube insertion (or 24 hours after admission if the patient is admitted at the unit with an NGT/NET); medications prescribed 120 hours after feeding tube insertion (or 120 hours after admission if the patient is admitted at the unit with an NGT/ NET); and medications prescribed 24 hours before patient discharge.

- Date of programmed feeding tube removal and the main reason; date and time of patient discharge; reason for patient discharge.

\section{Procedures}

Data collection started on February 2019 and will be completed in 24 months. At each centre, a registered nurse in the medical ward and a research assistant will serve as a liaison to the study investigators; a designated nurse coordinator will ensure that the collection of data will be carried out completely and correctly. The liaison nurse and the research assistant at each centre will attend a total of 16 hours of theoretical and practical formal training sessions led by the regional study coordinator, at which the overall study design will be presented and each electronic data collection forms will be explained. The study protocol will neither mandate nor direct any element of patient care and thus will pose no risk to patients.

A data collection guideline will be developed with the purpose of standardising data recording in the seven hospitals participating in the study. This guideline will consist of general information related to the research project; general instructions on how to access the electronic data collection forms using mobile devices; definition of each variable and additional information for completing the variables that make up the three electronic data collection forms. The guideline will be available on printed version and by email to assist all the research team on data collection.

Before data collection starts, the researchers will identify the patients who will start the use of an NGT/NET during hospitalisation, according to the inclusion criteria proposed for this study. In Brazilian hospitals, NGT/NET are inserted by registered nurses. 
Researchers will explain the research objectives and will ask patients, or their legal guardians, to voluntarily sign the Informed Consent Form.

For purposes of confidentiality, each patient will be identified by a unique identifying number; data will be collected from the moment a patient starts using an NGT/NET until the patient is discharged (due to death or non-death).

Using a mobile device, research assistants will collect demographic data at patient admission, and prospectively record clinical and therapeutic data from the patients' medical record. Considering that four hospitals participating in this study have present electronic medical records, these will be accessed in the computers available in the medical wards/units. In both situations, the researchers will request permission from the nurse responsible for the unit to access the computers and to record the information in the electronic data collection forms. The consultation of the medical records will occur in the afternoon or at night because these are the periods with the least movement of people.

\section{Assessment of the complexity of patients with NGT/NET}

Patient complexity will be assessed by an experienced nurse, member of the research team, on the first day of feeding tube use, on the day of the incident and weekly until patient discharges. Nurses will have up to 24 hours to complete their evaluation.

For this purpose, the PCS proposed by Fugulin ${ }^{35}$ will be used. The PCS is recommended by the Federal Nursing Council, Brazil, ${ }^{37}$ and it was developed to classify patients according to the degree of dependence of the nursing team. This instrument has nine critical indicators: mental state, oxygenation, vital signs, motility, ambulation, feeding, body care, elimination and therapeutics. Therefore, the scores are distributed in five categories that correspond to the complexity of the assistance: minimum care (scores of 9-14), intermediate care (scores of 15-20), high dependency (scores of 21-26), semi-intensive (scores of 27-31) and intensive care (scores $>31$ ).

\section{Assessment of the severity of comorbid diseases of patients with NGT/NET}

Regarding the severity of comorbid diseases, this will be evaluated on admission at the medical ward/unit, on the first day of use of the short-term feeding tube, on the day of the incident and weekly until patient discharges. For this purpose, the $\mathrm{CCI}^{38}$ will be used. The CCI is the most widely used comorbidity, which is a method of categorising patients' comorbidities, according to the ICD. The aim of the CCI is to measure the severity of the patient, regardless of the main diagnosis, and therefore, to predict the 1-year mortality of patients. The final score is the result of the sum of the weights assigned to the comorbidities recorded as secondary diagnoses; the higher the score, the greater the risk of the patient dying. It should be noted that the CCI will be adjusted according to the patient's age, so that, from the age of 50 , a point will be added to the final score for every decade of life. ${ }^{38}$

Based on the final CCI score, patients will be divided into three groups: mild (with CCI scores of 1-2), moderate (with CCI scores of 3-4) and severe (with CCI scores $\geq 5$ ). In addition, survival time in days will be calculated from the first day patient starts using a feeding tube during hospital stay to the death of a patient. ${ }^{39}$

\section{Assessment of the NGT/NET-related incidents}

In this study, incident was defined as an event or circumstance that could have resulted, or did result, in unnecessary harm to a patient. ${ }^{36}$ The NGT/NET-related incidents considered in this study are as follows:

- Mechanical incident (unplanned/accidental removal; obstruction; displacement/migration; epistaxis; nasal mucosa oedema; perforation or stenosis of the oesophagus; perforation of the brain; pneumothorax; various attempts to introduce the tube).

- Gastrointestinal incident (nausea/vomiting; diarrhoea; constipation; colic/abdominal distension/ flatulence).

- Infection (aspiration pneumonia; gastroenterocolitis).

- Other incidents (skin lesion associated with the NGT/ NET insertion; bronchoaspiration; wrong connection; quality of the tube material).

Based on previous studies, ${ }^{41}$ three methods will be used for incidents detection:

1. Prompted spontaneous reporting: healthcare professionals (nursing and medical staff) and patients/ caregivers will be continuously prompted to report any NGT/NET-related incidents to the investigators. A pen and a booklet containing instructions on incident reporting will be available and remain at the bedside of each patient participating in the study.

2. Researchers will visit the wards/units at least twice a week to request information about the incidents, along with healthcare professionals (nursing and medical staff) and patients/caregivers.

3. Researchers will review the booklet and medical records at least twice a week to obtain information about NGT/NET-related incident.

Using a mobile device, the research assistant will record data related to the incidents in the electronic data collection form developed for this purpose. More than one incident may occur on the same day and/or at the same time (ie, tube obstruction and unplanned removal), thus each incident will be recorded on a different form, resulting in a form filled for each incident.

\section{Assessment of pDDls in patients with NGT/NET}

Regarding the analysis of pDDIs, it will be performed only in patients with a hospital stay of at least 5 days because longer hospital stays are associated with the likelihood of adverse drug events. ${ }^{42}$

The research assistants will record on electronic data collection forms all medications and enteral nutrition prescribed for patients, regardless of whether they were 
administered or not. Data related to the medications will be collected in the following moments of hospitalisation: at admission; 24 hours and 120 hours after admission because it is the period of greatest therapeutic adjustment. ${ }^{43-45}$ Data related to medications will also be recorded 24 hours before patient discharge and on the day of the incident. Data related to enteral nutrition will be recorded on admission and on the day of the incident.

Medications will be classified according to the WHO Chemical Anatomical Therapeutic Classification (ATC), ${ }^{46}$ and the analysis of drug interactions will be performed based on drug monographs from the Drug Reax System database from Thomson Healthcare because it is a highly reliable software. ${ }^{47}$ The software is also available in the journal portal of the Coordination of Improvement of Higher-Level Personnel-CAPES, Brazil. pDDIs will be classified according to the speed of action, documentation and severity, as recommended by Drug Reax.

\section{Data analysis}

Data will be downloaded from the Survey Monkey to a computer file and analysed using the SPSS v. 25.0. In the statistical analysis, we will apply calculation of proportions and measures of central tendency and variability. Quantitative variables will be assessed for normal distribution using the Kolmogorov-Smirnov test. The importance of the normal distribution evaluation is justified, among others, by the subsequent indication of parametric and non-parametric statistical tests. Pearson's chi-square $\left(\chi^{2}\right.$ and Fisher's exact) tests will be used to compare the response variables with the categorical explanatory variables, when indicated. For the comparison of the variable responses with the quantitative explanatory variables, $t$ or Mann-Whitney tests will be performed, when indicated.

To examine the predictive role of CCI on mortality of patients having an NGT/NET, a one-way analysis of variance will be used to compare group differences stratified by CCI, and otherwise, chi-square tests will be applied. All analysis will be carried out considering a significance level of $5 \%(\alpha=0.5)$.

\section{Patient and public involvement}

Patients were not involved in the setting of the research questions or the outcome measures. However, we have specific plans to disseminate the results of the research to the patient community through social media (ie, Facebook, Twitter), print media (ie, Patient Safety Alert), the internet (ie, links to study reports on the Patient Safety Research Group website) and community/stakeholder engagement activities (ie, community forums, stakeholder meetings).

\section{ETHICS AND DISSEMINATION}

The study was approved by the Research Ethics Committee of the University of São Paulo at Ribeirão Preto College of Nursing, according to the Resolution No. 466/2012, of the National Council of Ethics in Research of the
Brazilian Ministry of Health, which addresses research ethics with humans (CAAE: 56166016.1001.5393). Written informed consent will be obtained from each patient, or their guardian, prior to enrolment in the study. Participants will be informed of the project aims and asked to voluntarily sign the consent form. They will be informed of possible risks which are discomfort or embarrassment when answering questions about NGT/ NET-related incidents, thus participants will be able to refuse to answer any question that they feel inconvenient or inappropriate, without causing harm to their care. No personally identifiable information will be collected in order to maintain the anonymity of the participants and they will be informed that the results will be used for possible publications.

The results will be reported to the hospitals and the findings may contribute to the hospital's policies and procedures to reduce the risks for NGT/NET-related incidents and to improve the quality of care and patient safety. There is very little information about NGT/NET-related incidents in developing countries, so this study will provide important information about risk levels. Results will also be reported through academic journals and conference presentations, social media (ie, Facebook, Twitter), print media (ie, Patient Safety Alert), the internet (ie, links to study reports on the Patient Safety Research Group website) and community/stakeholder engagement activities (ie, community forums, stakeholder meetings). The project team includes academic researchers, hospital clinicians and experts involved in patient safety. This provides the project with access to a range of other conduits through which to disseminate results to, for example, policy makers and system implementers.

\section{Author affiliations}

${ }^{1}$ General and Specialized Nursing, University of São Paulo at Ribeirão Preto College of Nursing, Ribeirao Preto, Sao Paulo, Brazil

${ }^{2}$ Nursing Department, Federal University of Acre, Rio Branco, Acre, Brazil ${ }^{3}$ Nursing Department, Ceará State University, Fortaleza, Ceará, Brazil

${ }^{4}$ Nursing Department, University of Santa Cruz do Sul, Santa Cruz do Sul, Rio Grande do Sul, Brazil

${ }^{5}$ Nursing Department, Faculty of Science and Technology of Campos Gerais, Campos Gerais, Minas Gerais, Brazil

${ }^{6}$ Nursing Department, University Paulista, Campinas, São Paulo, Brazil

${ }^{7}$ Psychiatric Nursing and Human Sciences, University of São Paulo at Ribeirão Preto College of Nursing, Ribeirão Preto, Brazil

Contributors FREG and MCAP initiated the project and are the chief investigators on the project; they made substantial contributions to the conception and design of the work. PRP, REFLC, JK, LMF, TCAT and AIM are associate investigators and all made significant contributions to the protocol in their specific areas of expertise. FREG prepared the first draft of this protocol and all authors have reviewed, provided input and gave final approval of the version published.

Funding The authors have not declared a specific grant for this research from any funding agency in the public, commercial or not-for-profit sectors.

Competing interests None declared.

Patient consent for publication Not required.

Ethics approval The study was approved by the Research Ethics Committee of the University of São Paulo at Ribeirão Preto College of Nursing (CAAE: 56166016.1001.5393).

Provenance and peer review Not commissioned; externally peer reviewed. 
Data sharing statement The datasets generated during and/or analysed during the study will be available from the corresponding author on reasonable request.

Open access This is an open access article distributed in accordance with the Creative Commons Attribution Non Commercial (CC BY-NC 4.0) license, which permits others to distribute, remix, adapt, build upon this work non-commercially, and license their derivative works on different terms, provided the original work is properly cited, appropriate credit is given, any changes made indicated, and the use is non-commercial. See: http://creativecommons.org/licenses/by-nc/4.0/.

\section{REFERENCES}

1. Gimenes FRE, Anacleto TA. Medication preparation and admininstration through feeding tubes and ostomy] [press release]. Belo Horizonte: Boletim ISMP Brasil, 2015.

2. Grant M, Martin S. Delivery of enteral nutrition. Advanced Practice in Acute \& Critical Care: AACN Clinical Issues, 2000.

3. Krenitsky J. Blind bedside placement of feeding tubes: treatment or threat? Practical Gastroenterology 2011:32-42.

4. Blumenstein I, Shastri YM, Stein J. Gastroenteric tube feeding: techniques, problems and solutions. World J Gastroenterol 2014;20:8505-24.

5. Figueredo LP. Complications of enteral nutritional therapy (NER) and associated factors in hospitalized patients. São Paulo: Universidade de São Paulo, 2011.

6. Stayner JL, Bhatnagar A, McGinn AN, et al. Feeding tube placement: errors and complications. Nutr Clin Pract 2012;27:738-48.

7. American Association of Critical-Care Nurses. AACH practice alert verification of feeding tube placement [press release]. Aliso Viejo (CA): AACN, 2009.

8. Amirlak B, Amirlak I, Awad Z, et al. Pneumothorax following feeding tube placement: precaution and treatment. Acta Med Iran 2012;50:355-8.

9. Arnau Alfonso JJ. [Methods for determining the correct location of a nasogastric tube after its insertion in adults patients]. Enferm Clin 2013;23:81-3.

10. Sorokin R, Gottlieb JE. Enhancing patient safety during feeding-tube insertion: a review of more than 2,000 insertions. JPEN J Parenter Enteral Nutr 2006;30:440-5.

11. Millin CJ, Brooks M. Reduce--and report--enteral feeding tube misconnections. Nursing 2010;40:59-60.

12. Metheny NA, Meert KL, Clouse RE. Complications related to feeding tube placement. Curr Opin Gastroenterol 2007;23:178-82.

13. Waitzberg D, Enck C, Miyahira N, et al. Nutrition Therapy: quality indicators. São Paulo: Project Guidelines; Brazilian Medical Association; Federal Council of Medicine, 2011.

14. Pillai JB, Vegas A, Brister S. Thoracic complications of nasogastric tube: review of safe practice. Interact Cardiovasc Thorac Surg 2005;4:429-33.

15. Gilbert RT, Burns SM. Increasing the safety of blind gastric tube placement in pediatric patients: the design and testing of a procedure using a carbon dioxide detection device. J Pediatr Nurs 2012;27:528-32.

16. Kuo YW, Yen M, Fetzer S, et al. Reducing the pain of nasogastric tube intubation with nebulized and atomized lidocaine: a systematic review and meta-analysis. J Pain Symptom Manage 2010;40:613-20.

17. Ferreira AM. Nasogastric and nasoenteric feeding tubes: how may the disconfort during placement be reduced?]. Rev Esc Enferm USP 2005;39:358-9.

18. Mitchell JF. Oral dosage forms that should not be crushed: Institute for Safe Medication Practices - ISMP, 2014.

19. van den Bemt PMLA, et al. Quality improvement of oral medication administration in patients with enteral feeding tubes. Quality and Safety in Health Care 2006;15:44-7.

20. Grissinger M. Preventing errors when drugs are given via enteral feeding tubes. P t 2013;38:575-6.

21. Simons SR, Abdallah LM. Bedside Assessment of enteral tube placement: aligning practice with evidence. Am J Nurs 2012;112:40-6.
22. Brazil. Strategic actions plan for coping with chronic noncommunicable diseases (CNCD) in Brazil 2011-2022. Brasília: Ministry of Health, Secretariat of Health Surveillance, Department of Health Situation Analysis, 2011.

23. World Health Organization. Global status report on noncommunicable diseases 2014. Geneva: World Health Organization, 2014.

24. Lord LM. Enteral Access Devices: Types, Function, Care, and Challenges. Nutr Clin Pract 2018;33:16-38.

25. Heydrich J, Heineck I, Bueno D. Observation of preparation and administration of drugs by nursing assistants in patients with enteral feeding tube. Brazilian Journal of Pharmaceutical Sciences 2009;45:117-20.

26. Emami S, Hamishehkar H, Mahmoodpoor A, et al. Errors of oral medication administration in a patient with enteral feeding tube. $J$ Res Pharm Pract 2012:1:37-40.

27. Pereira SRM, Coelho MJ, AMFd M, et al. Causes for the unplanned removal of the feeding tube in intensive care. Acta Paul Enf 2013;26:338-44.

28. Fisher C, Blalock B. Clogged feeding tubes: a clinician's thorn. In: Parrish CR, editor. Practical Gastroenterology 2014.

29. Palleria C, Di Paolo A, Giofrè C, et al. Pharmacokinetic drug-drug interaction and their implication in clinical management. $J$ Res Med Sci 2013;18:601-10.

30. Hoffmann B, Beyer M, Rohe J, et al. "Every error counts": a webbased incident reporting and learning system for general practice. Qual Saf Health Care 2008;17:307-12.

31. World Health Organization. Patient safety research: a guide for developing training programmes. Geneva: WHO, 2012.

32. Brazil. Ordinance No. 529, of April 1, 2013. Establishes the National Patient Safety Program (PNSP). Brasília, DF: Diário Oficial da União, 2013.

33. Brazil. Reference Document for the National Patient Safety Program. Brasilia: Ministry of Health, 2014.

34. World Health Organization. WHO Patient Safety. WHO patient safety research: better knowledge for safer care. Geneva: World Health Organization, 2009.

35. Fugulin F. Sizing of nursing staff: evaluation of the staffing of hospitalization units of a teaching hospital. São Paulo: School of Nursing, University of São Paulo, 2002.

36. World Health Organization. Conceptual framework for the international classification for patient safety. Geneva: World Health Organization, 2009.

37. Federal Nursing Council. Resolution COFEN N²93/2004. Rio de Janeiro: COFEN, 2004

38. Charlson ME, Pompei P, Ales KL, et al. A new method of classifying prognostic comorbidity in longitudinal studies: development and validation. J Chronic Dis 1987;40:373-83.

39. Huang YQ, Gou R, Diao YS, et al. Charlson comorbidity index helps predict the risk of mortality for patients with type 2 diabetic nephropathy. J Zhejiang Univ Sci B 2014;15:58-66.

40. Bates DW. The Costs of Adverse Drug Events in Hospitalized Patients. JAMA: The Journal of the American Medical Association 1997;277:307-11.

41. Bates DW, Cullen DJ, Laird N, et al. Incidence of adverse drug events and potential adverse drug events. Implications for prevention. ADE Prevention Study Group. JAMA 1995;274:29-34.

42. Strom BL, Kimmel SE, Hennessy S. Pharmacoepidemiology: Wiley, 2011.

43. Biswal S, Mishra P, Malhotra S, et al. Drug utilization pattern in the intensive care unit of a tertiary care hospital. J Clin Pharmacol 2006;46:945-51.

44. Lima RE, De Bortoli Cassiani SH. Potential drug interactions in intensive care patients at a teaching hospital. Rev Lat Am Enfermagem 2009;17:222-7.

45. Reis AM, de Carvalho RE, de Faria LM, et al. [Prevalence and clinical significance of interactions drug-enteral nutrition in Intensive Care Units]. Rev Bras Enferm 2014;67:85-90.

46. World Health Organization. ATC/DDD index 2018. Geneva: WHO, 2018.

47. Vonbach P, Dubied A, Krähenbühl S, et al. Evaluation of frequently used drug interaction screening programs. Pharm World Sci 2008;30:367-74. 